Louisiana State University

LSU Digital Commons

3-3-2020

\title{
Hyaluronic Acid-Cellulose Composites as Patches for Minimizing Bacterial Infections
}

\author{
Kelsey M. Lopez \\ Louisiana State University \\ Sudhir Ravula \\ Louisiana State University \\ Rocío L. Pérez \\ Louisiana State University \\ Caitlan E. Ayala \\ Louisiana State University \\ Jack N. Losso \\ Louisiana State University
}

See next page for additional authors

Follow this and additional works at: https://digitalcommons.Isu.edu/chemistry_pubs

\section{Recommended Citation}

Lopez, K., Ravula, S., Pérez, R., Ayala, C., Losso, J., Janes, M., \& Warner, I. (2020). Hyaluronic Acid-Cellulose Composites as Patches for Minimizing Bacterial Infections. ACS Omega, 5 (8), 4125-4132.

https://doi.org/10.1021/acsomega.9b03852

This Article is brought to you for free and open access by the Department of Chemistry at LSU Digital Commons. It has been accepted for inclusion in Faculty Publications by an authorized administrator of LSU Digital Commons.

For more information, please contact ir@lsu.edu. 


\section{Authors}

Kelsey M. Lopez, Sudhir Ravula, Rocío L. Pérez, Caitlan E. Ayala, Jack N. Losso, Marlene E. Janes, and Isiah M. Warner 


\title{
Hyaluronic Acid-Cellulose Composites as Patches for Minimizing Bacterial Infections
}

\author{
Kelsey M. Lopez, Sudhir Ravula, Rocío L. Pérez, Caitlan E. Ayala, Jack N. Losso, Marlene E. Janes, \\ and Isiah M. Warner*
}

Cite This: ACS Omega 2020, 5, 4125-4132

Read Online

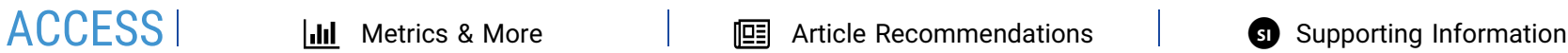

ABSTRACT: A facile method was used to synthesize biocomposites containing differing ratios of hyaluronic acid (HA) and cellulose (CEL). Based on the properties of the individual polymers, the resultant composite materials may have potentially great wound care properties. In the method outlined here, 1-butyl3-methylimidazolium chloride $([\mathrm{Bmim}][\mathrm{Cl}])$, a simple ionic liquid, was used as the sole solvent without chemical modifiers to dissolve the biopolymers at ratios of 1:1 and 2:1 HA to CEL. This method was completely recyclable since the ionic liquid, $[\mathrm{Bmim}][\mathrm{Cl}]$, can be recovered. Results from spectroscopic measurements [Fourier transform infrared (FT-IR) and X-ray diffraction (XRD)] confirm the interaction between $\mathrm{HA}$ and CEL. Scanning electron

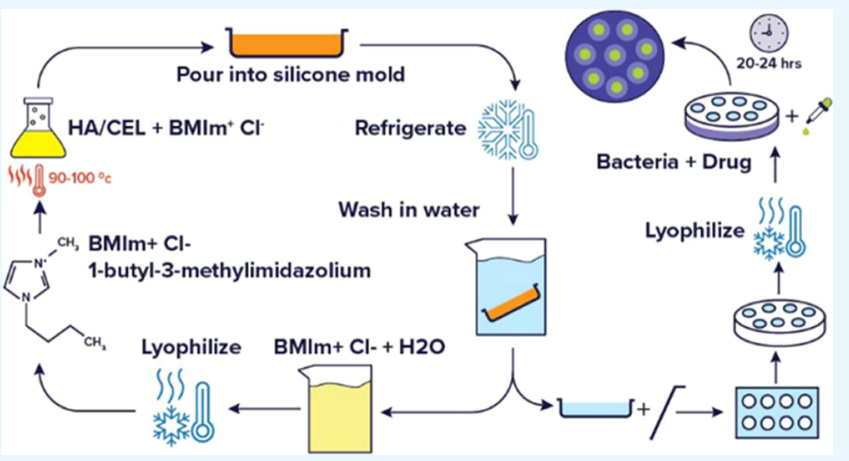
microscopy (SEM) images reflect differing biopolymer ratios and the resulting impact on the texture and porosity of these composite materials. The composites exhibited high swelling capacity in various media. These composites were also drug-loaded to examine drug release properties for greater potential in combating Staphylococcus aureus infections.

\section{INTRODUCTION}

Hyaluronic acid (HA) is a glycosaminoglycan that is found throughout connective, endothelial, and neural tissues. ${ }^{1}$ It has unique properties, such as high hygroscopicity, viscoelasticity, biocompatibility, and nonimmunogenicity, and does not generate toxic products upon degradation. ${ }^{2}$ These unique properties are thought to be beneficial in wound care bandage materials as HA has been investigated for a number of clinical applications, including lubrication and mechanical support of arthritic joints, ${ }^{3,4}$ as a surgical aid in ophthalmological surgery, ${ }^{5,6}$ drug delivery agent, ${ }^{7-9}$ and to facilitate surgical wound healing. ${ }^{10-12}$ Hyaluronic acid has also recently been used in skin-care products, such as facial moisturizers, as a result of its aqueous viscous consistency and nonallergenic tissue friendliness. ${ }^{13}$ However, one disadvantage of $\mathrm{HA}$ is its lack of adequate mechanical properties. ${ }^{14}$ To overcome this disadvantage, our laboratory focuses on the fabrication of composites from HA and cellulose (CEL) in this manuscript.

Cellulose (CEL) is a polysaccharide and the primary constituent of plant cell walls. It is biocompatible and biodegradable. Cellulose was chosen for this study because it lends chemical stability and mechanical strength to composite materials. ${ }^{15}$ Different forms of cellulose have recently been used as additives in composite biopolymer fabrication. For example, Huang et al. fabricated berberine-enriched carboxymethylcellulose-hyaluronic acid hydrogels with excellent viscosity to provide anti-inflammatory and antibacterial functions for minimizing postsurgical complications. However, these composites required polyvinyl alcohol as a base to assist membrane formation. ${ }^{16}$ Jia et al. have shown that bacterial cellulose (BC) and hyaluronic acid can be combined into composites with enhanced tensile strength and Young's modulus. ${ }^{17}$ While BC has been widely used for various applications, cellulose derived from cotton (as used in this manuscript) is thermodynamically more stable than $\mathrm{BC}^{18}$ In addition, the fabrication of these composites required crosslinking reactions or soaking $\mathrm{BC}$ for several days in $\mathrm{HA}^{17}$ In another example, Domingues et al. ${ }^{19}$ determined that cellulose nanocrystals and hyaluronic acid can be combined into hydrogel materials for injectable purposes. However, these materials also required the covalent modification of $\mathrm{HA}$ and CEL using additional cross-linkers. ${ }^{19}$

Tran et al. ${ }^{15}$ used ionic liquids (ILs) to combine chitosan and cellulose into composite materials with enhanced tensile strength, as well as antimicrobial properties. In addition, materials reported by Tran et al. exhibited an increased

Received: November 12, 2019

Accepted: February 10, 2020

Published: February 21, 2020 

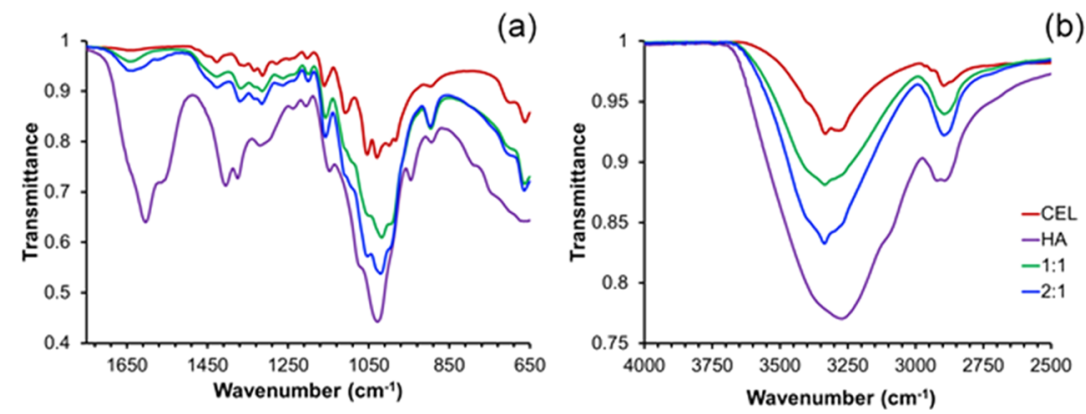

(b)

Figure 1. FT-IR spectra of CEL, HA, 1:1, and 2:1 composites (a), and $\mathrm{O}-\mathrm{H}$ stretching region (b).

antimicrobial effect on a wider range of bacteria than other chitosan-based materials. ${ }^{15,20,21}$ By fabrication of these biopolymers into composite materials, we rationalize that the combined properties of mechanical integrity, hygroscopicity, and biocompatibility could be beneficial for potential wound care devices. To obtain these composites, we hypothesize that the use of ionic liquids would be a viable and facile alternative for dissolving biopolymers, such as HA and CEL, as compared to previously mentioned procedures.

Ionic liquids (ILs) are organic salts that are liquid at temperatures below $100{ }^{\circ} \mathrm{C} .{ }^{22}$ ILs have various, unique chemical and physical properties, including high thermal stability, high solubility power, low volatility, and negligible vapor pressure. Because of these properties, ILs have been labeled as "green" alternatives to traditional, organic solvents. ${ }^{23}$ In addition, due to their high solvating ability, ILs have been used as solvents in dissolving biopolymers, including cellulose, extensively. ${ }^{15,24-27}$ However, limited information was found regarding ILs to dissolve HA. For this reason, we explored ILs as potential solvents for composite fabrication. In this study, we demonstrate that a simple ionic liquid, 1-butyl-3methylimidazolium chloride $([\mathrm{Bmim}][\mathrm{Cl}])$, solubilizes both hyaluronic acid and cellulose and allows composite formation without the use of chemical modifications or cross-linking reactions. One anticipated challenge of $[\mathrm{Bmim}][\mathrm{Cl}]$ is the high viscosity associated with the halide anion, ${ }^{28}$ but viscosity was not an issue with the elevated temperature needed for fabrication. Overall, this method proved that it is simple, green, and completely recyclable.

We also note that composites impregnated with antimicrobial agents may be used as topical medicated devices. Previous studies support the use of antimicrobial group of uniform materials based on organic salts (GUMBOS) as a chemical approach for combating various Gram-negative and Grampositive bacteria. ${ }^{29,30}$ GUMBOS are a group of novel materials, similar to ILs, composed of bulky inorganic and/or organic counterions with melting points between 25 and $250{ }^{\circ} \mathrm{C} .{ }^{31}$ GUMBOS employed in this manuscript were formulated from chlorhexidine, an antiseptic, and $\beta$-lactam antibiotics, oxacillin and cephalothin. These materials have been proven to be more effective than the constituent parent compounds against various strains of bacteria, most notably Staphylococcus aureus, which is one of the most common bacteria found on the skin and hair, as well as noses and throats of humans and animals. ${ }^{32}$ This bacterium is capable of manifesting severe clinical infections.

Herein, we explore the development and characterization of a green and recyclable method using ionic liquids to fabricate composites from biocompatible and biodegradable biopolymers (e.g., hyaluronic acid and cellulose) and exploit their biomedical use as wound care devices for minimizing bacterial infections. The main advantages of this approach are the ease of fabrication, cross-linking agents are not necessary, and high swelling abilities of resulting composites. Preparation of these biocomposites demonstrates promising applications as patches for use in wound care.

\section{RESULTS AND DISCUSSION}

FT-IR Analysis. FT-IR spectroscopy was employed to determine the chemical structure of the composite materials. Spectra were measured using a Bruker Tensor 27 (Billerica, MA) under transmission wavenumber ranging from 650 to $4000 \mathrm{~cm}^{-1}$ (Figure 1a,b). The HA spectrum had absorption bands at 1604 and $1405 \mathrm{~cm}^{-1}$. These are indicative bands of the carboxylate asymmetric stretching vibration and carboxylate symmetric stretching, respectively. ${ }^{33}$ Bands at 1145 and $1027 \mathrm{~cm}^{-1}$ are $\mathrm{C}-\mathrm{O}-\mathrm{C}$ stretching vibration of the HA skeleton. ${ }^{34}$ Two signals at 1560 and $1322 \mathrm{~cm}^{-1}$ are indicative of amide bands. ${ }^{33}$ In the region of $1150-650 \mathrm{~cm}^{-1}$, there is little difference between the spectra of the composites and HA. This verifies the presence of HA in these composite materials. All spectra have a strong absorption band between 3600 and $3000 \mathrm{~cm}^{-1}$, which is indicative of the $\mathrm{O}-\mathrm{H}$ stretching region (Figure $1 \mathrm{~b}$ ). While these peaks are not as broad in composite materials, they are broader than the cellulose $\mathrm{O}-\mathrm{H}$ peak. This narrow peak for cellulose is presumably due to hydrogen bonding within the glucose monomer network. This change in peak shape within the composites suggests hydrogen-bonding interactions between the $\mathrm{HA}$ and cellulose in the composite materials. $^{35}$

Thermal Stability of Composites. Figure 2 displays the thermogravimetric analysis (TGA) spectra for the parent biopolymers and composite materials. TGA was performed using a Hi Res modulated TGA 2950 thermogravimetric analyzer (TA Instruments, New Castle, DE). Small pieces weighing about $20 \mathrm{mg}$ were placed in a platinum pan and

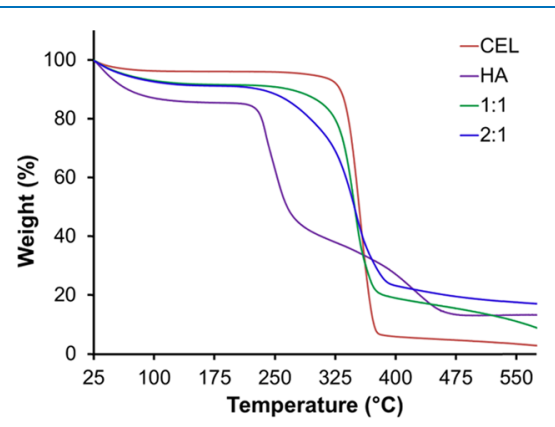

Figure 2. TGA spectra of cellulose, HA, and composites. 
heated from room temperature to $575{ }^{\circ} \mathrm{C}$ at a rate of $10{ }^{\circ} \mathrm{C} /$ min. Onset temperatures were extrapolated and denote the temperature at which the weight loss begins. Onset temperatures for cellulose and hyaluronic acid were 332.70 and $228.12{ }^{\circ} \mathrm{C}$, respectively (Table 1 ). Onset temperatures of the

Table 1. Onset Temperatures of the Composites and Parent Biopolymers

\begin{tabular}{lc}
\multicolumn{1}{c}{ compound } & onset temperature $\left({ }^{\circ} \mathrm{C}\right)$ \\
hyaluronic acid & 228.1 \\
cellulose & 332.7 \\
$1: 1$ composite & 324.3 \\
$2: 1$ composite & 305.4
\end{tabular}

composites decreased by 9 and $30^{\circ} \mathrm{C}$, as compared to those of cellulose for $1: 1$ and $2: 1$, respectively. The $1: 1$ composite had an onset temperature of $324.27{ }^{\circ} \mathrm{C}$, while the $2: 1$ composite had an onset temperature of $305.44{ }^{\circ} \mathrm{C}$. The $2: 1$ composite most likely had a lower degradation temperature due to the higher concentration of $\mathrm{HA}$, which is known to be a thermally sensitive material.

X-ray Diffraction (XRD). X-ray diffraction was employed to determine how the varying amount of HA affects the crystallinity of cellulose. XRD was performed using an Empyrean X-ray diffractometer (Malvern Panalytical, U.K.). Evaluation of data presented in Figure 3 a confirms that HA is completely amorphous. The supramolecular structure of fibrous cellulose exists as cellulose I, and its crystallinity is a result of a ratio between two forms $\left(\mathrm{I}_{\beta}\right.$ and $\left.\mathrm{I}_{\alpha}\right)$. As shown in Figure 3, fibrous cellulose has five characteristic peaks at 14.9, $16.4,20.6,22.7$, and $34.5^{\circ}$, which corresponds to the $101,10 \overline{1}$, 021,002 , and 040 planes, respectively. ${ }^{36,37}$ The crystallinity pattern of fibrous cellulose is more characteristic of a higher concentration of cellulose $\mathrm{I}_{\beta}$ than $\mathrm{I}_{\alpha}$, a ratio more prevalent in woody plants and cotton, which is the origin of cellulose used in this manuscript. ${ }^{18}$ In general, the composites maintain the characteristic peaks of cellulose. However, there is a slight increase in amorphous scattering between 14.9 and $22.7^{\circ}$ diffraction angles in both $1: 1$ and $2: 1$ composites.

Crystallinity indices (CIs) of these materials were determined using the Segal method (eq 2), whereby $I_{002}$ is the intensity of the 002-lattice diffraction and $I_{\mathrm{am}}$ is the intensity of diffraction at $2 \theta=18^{\circ} .{ }^{37}$ The crystallinity indices are presented in Table 2. According to eq 1 , the CI of cellulose is $85.9 \%$. As shown in Table 2, CIs of both composites were lower than that of cellulose with the $2: 1$ composite having the lowest CI. This can be attributed to the higher content of amorphous HA in relation to cellulose within the composite
Table 2. Crystallinity Indices (\%) of the Composites and Parent Biopolymers

\begin{tabular}{|cc}
\multicolumn{1}{c}{ materials } & crystallinity index (\%) \\
hyaluronic acid & N/A \\
cellulose & 85.9 \\
1:1 composite & 78.1 \\
2:1 composite & 76.6 \\
\hline
\end{tabular}

network. The amorphous material disrupts the crystalline lattice of the cellulose, thus lowering its CI

$$
\mathrm{CI} \%=\frac{I_{002}-I_{\mathrm{am}}}{I_{002}} \times 100 \%
$$

Drug loading of the composites was also evaluated using XRD (Figure S3). Moreover, powder XRD was employed to elucidate the crystalline nature of $[\mathrm{CHX}][\mathrm{Oxa}]$ and amorphous nature of $[\mathrm{CHX}][\mathrm{Ceph}]$ (Figure S4). There is no obvious difference between the XRD spectra of plain composites versus drug-loaded. However, there are slight changes in the crystallinity indices, which were calculated for drug-loaded composites (Table S1). [CHX][Oxa] had a larger effect on $\mathrm{CI}$; for example, in 1:1 composites, [CHX][Oxa] loading lowered the CI from 78.1 to $75.3 \%$. This could possibly be due to adsorption of the drug onto the surface of the materials. In contrast, with 2:1 composites, [CHX][Oxa] loading increased the CI from 76.6 to $77.9 \%$ as a result of greater interactions of $[\mathrm{CHX}][\mathrm{Oxa}]$ within the network of these composites.

Scanning Electron Microscopy (SEM). Composites were cut into small pieces, and the surfaces (before and after drug loading) were spin-coated with a thin layer of conductive platinum. Surface morphologies were evaluated by scanning electron microscopy (Quanta 3D DualBeam FEG FIB-SEM, FEI) at $5 \mathrm{kV}$. All images (Figure 4) are at 5000×. Highermagnification images can be found in the Supporting Information (Figures S5 and S6). The composites exhibited a textured, porous surface; however, for a 2:1 composite, the pores were larger with more irregularity in shape. The fibrous networks of CEL and HA are clearly delineated within the pores. Instead, the 1:1 composite exhibited a smoother, planar texture. In comparison to the original materials, drug-loaded composites also exhibited a smoother-looking surface, indicating loading of the drug on the surface and in the pores. As a result, the fibrous networks were no longer observable after drug loading of $[\mathrm{CHX}][\mathrm{Ceph}]$ in both $1: 1$ and 2:1 composites. It is presumed that the loading of [CHX][Oxa] would also exhibit the same smooth-looking surface.
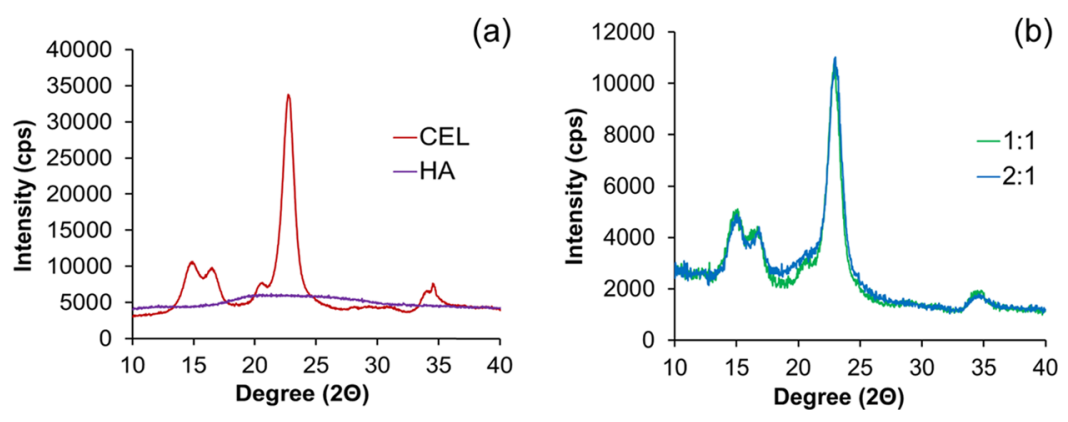

Figure 3. XRD spectra of CEL and HA (a), and 1:1 and 2:1 composites (b). 


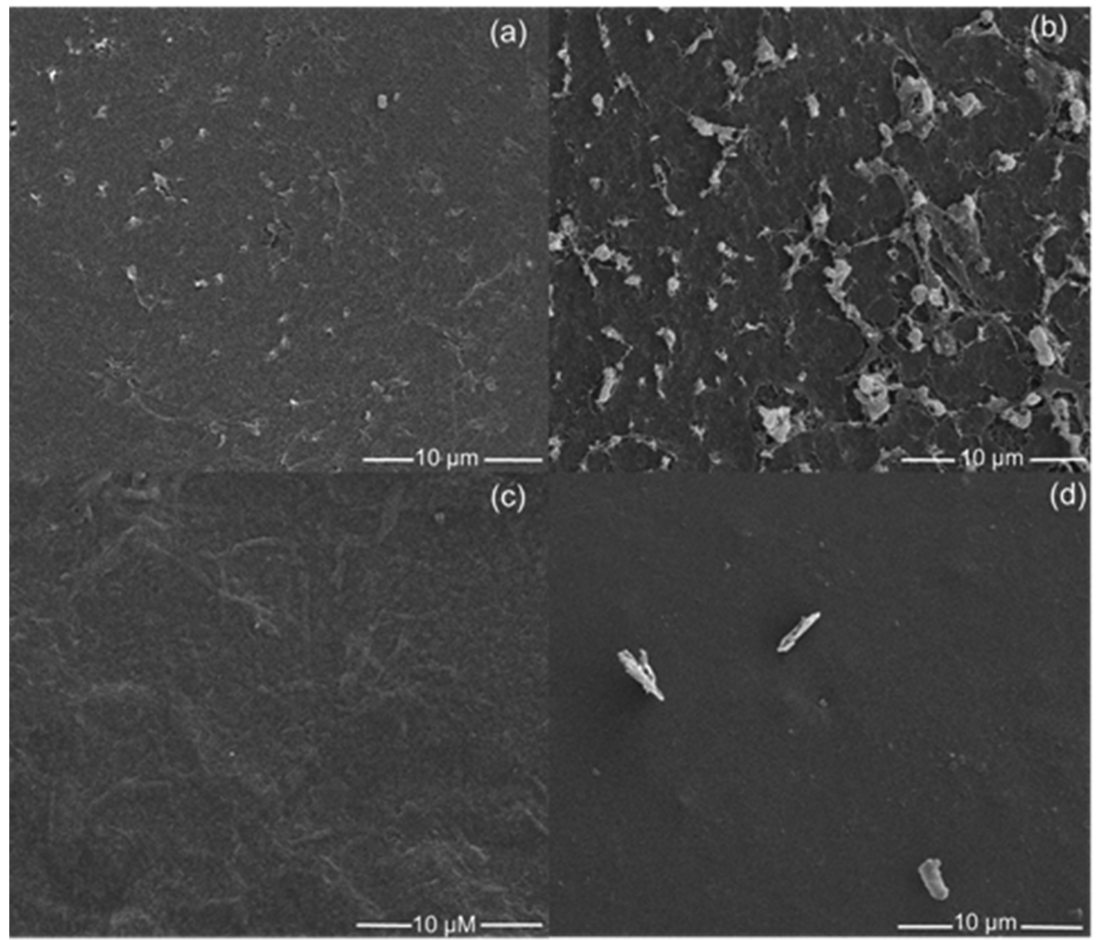

Figure 4. Morphology of 1:1 composite (a), 2:1 composite (b), [CHX][Ceph]-loaded 1:1 composite (c), and [CHX][Ceph]-loaded 2:1 composite (d).

Cross-sectional areas (before and after drug loading) were also evaluated by SEM at $5 \mathrm{kV}$. These images can be found in the Supporting Information (Figures S7 and S8). Crosssectional areas of composite materials (1:1 and 2:1) show no discernable difference between the drug-loaded and nondrugloaded composites. However, 1:1 composites exhibited a network of higher density within the matrix as compared to $2: 1$ composites. Similar to the surface, 2:1 composites exhibited a much more porous network within the composite matrix.

Swelling Studies. Swelling studies were performed to demonstrate the exudation capacity of these potential wound care composites, as well as investigate the effect of varying polymer ratios on capacity. Swelling percentages are illustrated in Figure 5. In all studied media, the 2:1 (HA/CEL) composite had a much higher swelling than the 1:1 (HA/CEL) material. These results indicate that the swelling ratio increased as the pore size and amorphous nature of the composites increased as amorphous regions are able to absorb more water than crystalline regions. ${ }^{38}$ Moreover, evaluation of these results indicates that as the density of fibers within the composite

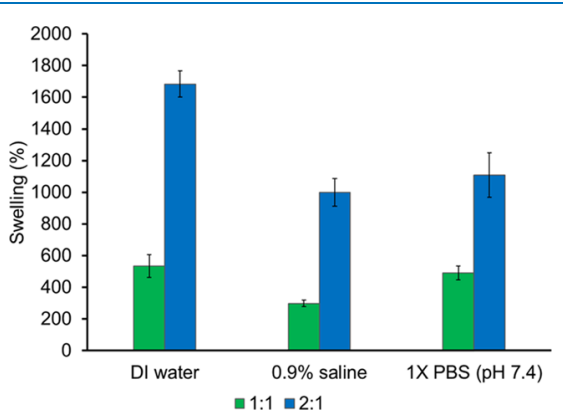

Figure 5. Swelling of composite materials in deionized water, $0.9 \%$ saline, and $1 \times$ phosphate-buffered saline (PBS) $(\mathrm{pH} 7.4)$. material decreased, the swelling increased. The high-density network within 1:1 composites could prevent the absorption of media, whereas the higher porosity within 2:1 materials provides voids for more facile absorption. The higher ratio of hygroscopic HA could also play a role in the absorption capacity of 2:1 composites. A larger degree of swelling could also be attributed to the higher concentration of sodium ions on HA that may have aided in balancing the internal osmosis. ${ }^{39}$ Interestingly, all composites maintained structural integrity, which can be attributed to the mechanical strength of cellulose. Images of dry and swollen composites are found in the Supporting Information (Figures S9 and S10). In comparison to hydrogels fabricated by Domingues et al., the swelling of composites synthesized in this manuscript was significantly higher. ${ }^{19}$ This could be due to no cross-linking agents used in the fabrication process. Instead of creating physical cross-links, this process is more akin to solution blending, thereby maintaining the hygroscopic functional groups of HA. Fibrous cellulose also has relatively higher absorbent properties, which could make it a better choice for topical applications such as wound management. ${ }^{40}$

Release Properties. The release of two antimicrobial GUMBOS was determined spectrophotometrically in a $0.9 \%$ saline solution at $\lambda=231 \mathrm{~nm}$. The cumulative amount of drug released and the percentage of drug released as a function of time for $[\mathrm{CHX}][\mathrm{Ceph}]$ and $[\mathrm{CHX}][\mathrm{Oxa}]$ are shown in Figures 6 and 7, respectively. Upon the placement of composites into the saline medium, an initial large amount of drug is released in what is known as "burst release". ${ }^{41}$ After the initial burst, the rate at which drugs are released decreases and all drugs have been released between 4 and $6 \mathrm{~h}$. This is evident from the stable plateau between the 4 and $6 \mathrm{~h}$ time point. Burst release could be beneficial for wound treatment as quick release could ensure the rapid reduction of bacteria from wound sites. ${ }^{41-43}$ Overall, the 1:1 composite showed a much higher release of 
(a)

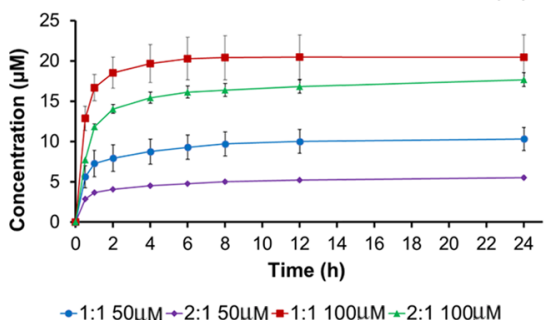

(b)

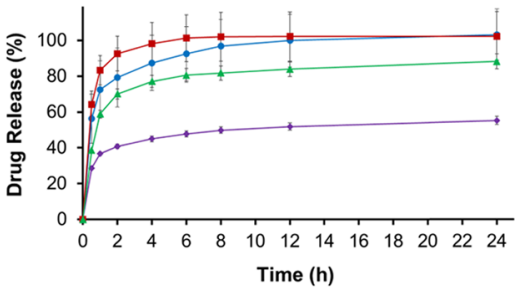

Figure 6. Cumulative drug release (a) and percent drug release (b) for $[\mathrm{CHX}][\mathrm{Ceph}]$.

(a)

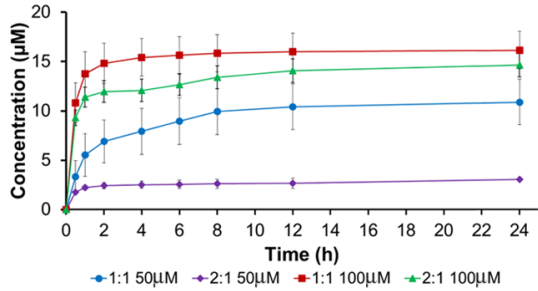

(b)

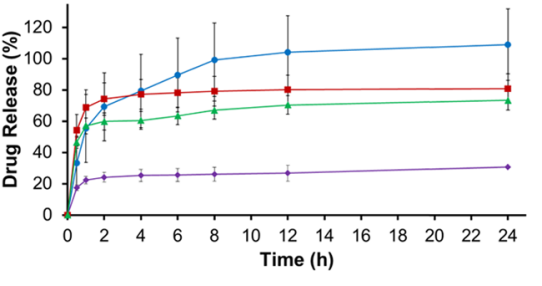

Figure 7. Cumulative drug release (a) and percent drug release (b) for [CHX][Oxa].

drugs than the 2:1 ratio. In both drug release studies, the 1:1 composite exhibited quantitative drug release as shown in Figures $6 \mathrm{~b}$ and $7 \mathrm{~b}$. In contrast, the $2: 1$ material did not achieve quantitative release over the same time period. This could be attributed to the morphology of the surface and inner network of the composites. The 1:1 composite, as seen in Figure 4a, exhibits less pores than the 2:1 composite and a denser inner network. Presumably, drug molecules are not physically able to penetrate the 1:1 network as deeply and concentrate more on the surface. In effect, these drug molecules are then released from the surface in a "burst" manner when placed into saline. In contrast, the 2:1 composite exhibited a much more porous surface (Figure 4b) and less dense inner network, which could allow trapping of the drug further within the fibrous network and impede the rate and quantity of the release of organic molecules. Both composites (1:1 and 2:1) released more $[\mathrm{CHX}][\mathrm{Ceph}]$ than $[\mathrm{CHX}][\mathrm{Oxa}]$, which we hypothesize could be a result of $[\mathrm{CHX}][\mathrm{Oxa}]$ having a stronger interaction with the composite material than $[\mathrm{CHX}][\mathrm{Ceph}]$.

Drug-loaded and drug-released composites were also evaluated using electron-dispersive X-ray spectroscopy (EDS) to confirm drug release (Quanta 3D DualBeam FEG FIB-SEM with an EDAX Pegasus EDS/EBSD detector). GUMBOS structures contain sulfur atoms (Figure S11). EDS spectra of plain composites can be found in the Supporting Information (Figures S12 and S13). After evaluation of drug-loaded composites using EDS, it was confirmed that the sulfurcontaining drugs were present in the loaded samples. We also observed that the sulfur signal decreases in drug-released samples (Figures S14-S16).

Disk Diffusion. Kirby-Bauer disk diffusion is a qualitative susceptibility test used to determine the sensitivity or resistance of a microorganism toward an antimicrobial agent. $^{44}$ In this study, disk diffusion was performed to demonstrate drug release from composites onto an agar surface. Three concentrations were loaded onto the disks and composite rounds: 10,50 , and $250 \mu \mathrm{M}$. During incubation, the drug diffuses from composites and disks into the agar. After 20-24 h incubation, zones of inhibition (ZOI) formed around the disks and composites, and the diameters were measured using a ruler. The zone of inhibition is a circular area around the disk (or composite) in which no bacteria has grown due to susceptibility of the bacterium to the drug. The rate of drug diffusion through agar can be governed by many different factors such as relative hydrophobicity, aqueous solubility, molecular weight, and intrinsic resistance of the microorganism. ${ }^{45}$ These ZOI values confirm that drug release occurred from the composites into the agar. The 1:1 composite loaded with $[\mathrm{CHX}][\mathrm{Ceph}]$ had larger zone diameters than the 2:1 composite of the same drug (Table 3). However, an

Table 3. Zone Diameters of Composites and Standard Disks for Diffusion of $[\mathrm{CHX}][\mathrm{Ceph}]$

\begin{tabular}{cccc} 
& \multicolumn{3}{c}{ zones of Inhibition $(\mathrm{mm} \pm$ standard deviation $)$} \\
\cline { 2 - 4 } concentration $(\mu \mathrm{M})$ & $1: 1$ composite & $2: 1$ composite & standard disk \\
10 & $15.7 \pm 1.2$ & $14.7 \pm 0.6$ & $17.6 \pm 1.1$ \\
50 & $27.3 \pm 1.5$ & $25.7 \pm 0.6$ & $28.2 \pm 1.0$ \\
250 & $36.3 \pm 2.5$ & $34.7 \pm 1.5$ & $37.3 \pm 1.0$
\end{tabular}

opposing trend was seen for zone diameters of $[\mathrm{CHX}][\mathrm{Oxa}]$ (Table 4). Overall, [CHX][Oxa] produced larger ZOIs versus $[\mathrm{CHX}][\mathrm{Ceph}]$. This could be due to the higher susceptibility of $S$. aureus to $[\mathrm{CHX}][\mathrm{Oxa}]$ as the standard disks impregnated with $[\mathrm{CHX}][\mathrm{Oxa}]$ produced larger zone diameters than $[\mathrm{CHX}][\mathrm{Ceph}]$. Moreover, it has been proven in previous studies that the water solubility of $[\mathrm{CHX}][\mathrm{Oxa}]$ is higher than that of $[\mathrm{CHX}][\mathrm{Ceph}]$, which allowed for better diffusion of

Table 4. Zone Diameters of Composites and Standard Disks for Diffusion of $[\mathrm{CHX}][\mathrm{Oxa}]$

\begin{tabular}{cccc} 
& \multicolumn{3}{c}{ zones of Inhibition $(\mathrm{mm} \pm$ standard deviation $)$} \\
\cline { 2 - 4 } concentration $(\mu \mathrm{M})$ & $1: 1$ composite & $2: 1$ composite & standard disk \\
10 & $21.3 \pm 0.6$ & $23 \pm 0.0$ & $19.2 \pm 3.2$ \\
50 & $31.7 \pm 0.6$ & $36.3 \pm 0.6$ & $34.2 \pm 1.8$ \\
250 & $38.3 \pm 1.2$ & $41.7 \pm 2.9$ & $42.2 \pm 1.7$
\end{tabular}


this drug through agar. ${ }^{46}$ Evaluation of data shows that the porous nature of these biopolymer composites allows for drug release, and this method of drug delivery has the potential for minimizing staph infections. Representative images of incubated agar plates with drug-loaded composites and standard disks can be found in the Supporting Information (Figure S17).

\section{CONCLUSIONS}

In summary, hyaluronic acid/cellulose-based composites were developed by the dissolution of biopolymers in $[\mathrm{Bmim}][\mathrm{Cl}]$, an ionic liquid, with no chemical modification. These composites were developed at two ratios (1:1 and 2:1 HA to $\mathrm{CEL}$ ) and showed a variety of morphological and structural changes that may prove applicable for wound care devices. Since IL can be removed from the composite material through washing with water and recovered by lyophilization, this method is also recyclable. Several spectroscopic and imaging techniques, including FT-IR, TGA, XRD, and SEM, were used for characterizing the composite material and monitoring $[\mathrm{Bmim}][\mathrm{Cl}]$ recovery. These composites showed great swelling capacity with the 2:1 composite exhibiting a much higher capacity. This could translate into an ability to absorb more wound exudate.

By loading with antimicrobial GUMBOS, these composites demonstrated their use as possible medicated devices for use in wound care. When both composites were drop-casted with the drug, there was uniform coverage on both materials. In drug release studies, burst release was seen from these composite materials with a higher release of the drug from 1:1 composite. Examination of results of in vitro disk diffusion tests showed that both composites allowed the diffusion of the drug into the medium for combating $S$. aureus infections. These results, in combination with inherent biocompatibility, strongly suggest potential biomedical applications of these composites. We will further investigate (1) the incorporation of drug materials within the composite network and (2) in vivo wound-healing properties of these composites using animal models.

\section{CHEMICALS AND MATERIALS}

Cellulose (medium, fibrous from cotton linters), silver nitrate, oxacillin sodium salt, and cephalothin sodium salt were obtained from Sigma-Aldrich. Hyaluronic acid sodium salt (1.5-2.2 $\mathrm{MDa})$ and chlorhexidine diacetate were obtained from Acros Organics. 1-Chlorobutane was obtained from Alfa Aesar. Methylimidazole was obtained from TCI Chemicals. S. aureus (ATCC 29213) was grown in brain heart infusion (BHI) broth and subcultured on mannitol salt agar. Inoculates were prepared in BHI and spread on Mueller-Hinton agar. All growth media was obtained from Accumedia.

\section{EXPERIMENTAL SECTION}

Synthesis and Characterization of HA/Cellulose Composite. Composites were fabricated in various ratios $(1: 1,1: 2,2: 1,1: 4$, and 4:1) of hyaluronic acid to cellulose and did not exceed 5\% w/w HA. However, 1:1 and 2:1 (HA/CEL) yielded the most stable composites that did not wash away upon the removal of $[\mathrm{Bmim}][\mathrm{Cl}]$; thus, this manuscript focuses only on these two ratios. Composites were fabricated by first dissolving sodium hyaluronan $(5 \% \mathrm{w} / \mathrm{w})$ in [Bmim]$[\mathrm{Cl}]$ and were synthesized according to procedures reported in the literature by Crowhurst et al. ${ }^{47}$ The solution was stirred in a vial at $90-100{ }^{\circ} \mathrm{C}$ in a mineral oil bath. Once all HA was dissolved, CEL ( 2.5 or $5 \% \mathrm{w} / \mathrm{w}$ ) was added in $10 \%$ increments to the vial. After CEL dissolved and the solution homogenized, the mixture was transferred to a silicone mold and placed in a refrigerator overnight to allow gel formation. The ionic liquid, $[\mathrm{Bmim}][\mathrm{Cl}]$, was recovered by soaking the composite in deionized water. Water was subsequently removed from $[\mathrm{Bmim}][\mathrm{Cl}]$ by lyophilization. Confirmation of $[\mathrm{Bmim}][\mathrm{Cl}]$ removal was concluded using FT-IR characterization and is detailed in Figure $\mathrm{S} 1$ in the Supporting Information. Silver nitrate $\left(\mathrm{AgNO}_{3}\right)$ ion test was also performed to confirm the removal of all $[\mathrm{Bmim}][\mathrm{Cl}]$ from the composite. These composites were cut to size $(1.27 \mathrm{~cm} \times 1.27 \mathrm{~cm})$, lyophilized overnight, and stored on the benchtop. Most notably, no crosslinking agents or chemical modifications were used in this synthesis. These HA/CEL-based composites were then characterized by Fourier transform infrared (FT-IR) spectroscopy, thermogravimetric analysis (TGA), X-ray diffraction (XRD), and scanning electron microscopy (SEM). They were also evaluated for swelling capacity and drug release properties.

Synthesis and Characterization of ChlorhexidineBased GUMBOS. Chlorhexidine-di cephalothin ([CHX]$[\mathrm{Ceph}])$ and chlorhexidine-di oxacillin ([CHX][Oxa]) GUMBOS were synthesized by ion-exchange procedures similar to those reported in the literature. ${ }^{30}$ Antibacterial susceptibility to various multidrug-resistant Gram-negative and Gram-positive bacteria, as well as cell viability, has been previously reported by Cole et al. ${ }^{30}$ These GUMBOS have also been shown to have increased pharmacokinetic properties, as well as increased intestinal bioavailability. ${ }^{30}$

Swelling Capacity. Dried composites were weighed and soaked in various media, such as $0.9 \%$ saline solution, distilled water, and $1 \times$ PBS buffer ( $\mathrm{pH} 7.4)$, at room temperature for up to $3 \mathrm{~h}$. Weights of the wetted composites were measured at defined intervals for up to $3 \mathrm{~h}$. Prior to weighing, filter paper was wiped across the surface of the composite to remove excess water. The swelling percent $(S \%)$ was determined using the following equation

$$
S \%=\frac{W_{\mathrm{s}-} W_{\mathrm{d}}}{W_{\mathrm{d}}} \times 100 \%
$$

In eq $2, W_{\mathrm{d}}$ is the weight of the dried composite and $W_{\mathrm{s}}$ is the weight of the respective swollen composite. The swelling percent is defined as the fractional increase in the composite weight due to absorption. All swelling studies were performed in triplicate.

Drug Loading. The drugs $[\mathrm{CHX}][\mathrm{Ceph}]$ and $[\mathrm{CHX}]$ [Oxa] were dissolved in methanol and diluted to the desired concentration in $500 \mu \mathrm{L}$ of methanol. Fifty microliters of the solution was drop-casted repeatedly onto the composite with the evaporation of methanol between each loading. It is presumed that drug loading is $100 \%$. The composite was then dried overnight using evaporation to ensure complete removal of methanol.

Drug Release. Composites were placed in vials with $5 \mathrm{~mL}$ of a $0.9 \%$ saline solution and were constantly shaken using a VWR S-500 orbital shaker at room temperature. At fixed time intervals, $4 \mathrm{~mL}$ of a $0.9 \%$ saline solution was removed and replaced with an equal volume of fresh saline to maintain a constant volume. The amount of drug released was assayed using a Shimadzu UV-3101PC UV-vis-near-infrared (NIR) 
spectrophotometer (Shimadzu Europe) at $\lambda=231 \mathrm{~nm}$. All drug release studies were performed in triplicate.

Kirby-Bauer Disk Diffusion. Testing was performed according to the Clinical and Laboratory Standards Institute (CLSI) recommended procedures. ${ }^{48}$ Kirby-Bauer disk diffusion assays ${ }^{44,45}$ were performed on $S$. aureus (ATCC 29213) to qualitatively demonstrate that therapeutic agents can be released from these dry composites and thus could minimize potential bacterial infections. Before lyophilization, composites were molded with a $7 \mathrm{~mm}$ plastic straw. These rounds were freeze-dried overnight and subsequently impregnated with therapeutic agents. Controls containing the same concentration of therapeutic agents were prepared using standard $6 \mathrm{~mm}$ paper disks to effectively compare the bacterial control relative to the composite. $S$. aureus was grown in a brain heart infusion (BHI) medium for $24 \mathrm{~h}$ and subcultured onto mannitol salt agar. Inoculum matching a $1.0 \mathrm{McF}$ arland standard was prepared in the BHI medium and spread evenly over the surface of a Mueller-Hinton agar plate to allow a confluent lawn of growth. Composite rounds and disks were placed on the agar plate and incubated upside down for 20-24 $\mathrm{h}$ at $37^{\circ} \mathrm{C}$. After incubation, zones of inhibition had formed around the composites and standard disks. Zone diameters were measured using a ruler. All experiments were performed in triplicate.

\section{ASSOCIATED CONTENT}

\section{SI Supporting Information}

The Supporting Information is available free of charge at https://pubs.acs.org/doi/10.1021/acsomega.9b03852.

Additional FT-IR spectra (Figures S1 and S2), XRD spectra (Figures S3 and S4), SEM images (Figures S5S8), composite images (Figures S9 and S10), structures of GUMBOS (Figure S11), EDS spectra (Figures S12S16), and disk diffusion image (Figure S17) (PDF)

\section{AUTHOR INFORMATION}

\section{Corresponding Author}

Isiah M. Warner - Department of Chemistry, Louisiana State University, Baton Rouge, Louisiana 70803, United States; ○ orcid.org/0000-0002-5336-7653; Phone: +1 225-5782829; Email: iwarner@lsu.edu; Fax: +1 225-578-3458

\section{Authors}

Kelsey M. Lopez - Department of Chemistry, Louisiana State University, Baton Rouge, Louisiana 70803, United States

Sudhir Ravula - Department of Chemistry, Louisiana State University, Baton Rouge, Louisiana 70803, United States; Department of Oral and Craniofacial Biology, School of Dentistry, Louisiana State University Health Science Center, New Orleans, Louisiana 70119, United States; 이이.org/ 0000-0002-3457-7102

Rocío L. Pérez - Department of Chemistry, Louisiana State University, Baton Rouge, Louisiana 70803, United States

Caitlan E. Ayala - Department of Chemistry, Louisiana State University, Baton Rouge, Louisiana 70803, United States

Jack N. Losso - Department of Food Sciences, Louisiana State University, Baton Rouge, Louisiana 70803, United States

Marlene E. Janes - Department of Food Sciences, Louisiana State University, Baton Rouge, Louisiana 70803, United States

Complete contact information is available at:

https://pubs.acs.org/10.1021/acsomega.9b03852

\section{Notes}

The authors declare no competing financial interest.

\section{ACKNOWLEDGMENTS}

The authors gratefully acknowledge financial support through NASA cooperative agreement NNX 16AQ93A under contract number NASA/LEQSF (2016-19)-Phase 3-10 and the National Science Foundation under Grant No. CHE-1905105. Any opinions, findings, and conclusions or recommendations expressed in this material are those of the author(s) and do not necessarily reflect the views of the National Science Foundation. The use of the LSU Shared Instrumentation Facility is also acknowledged.

\section{REFERENCES}

(1) Ivanova, E. P.; Bazaka, K.; Crawford, R. J. Natural Polymer Biomaterials: Advanced Applications. New Functional Biomaterials for Medicine and Healthcare; Woodhead Publishing, 2014; pp 32-70.

(2) Necas, J.; Bartosikova, L.; Brauner, P.; Kolar, J. Hyaluronic acid (hyaluronan): a review. Vet. Med. 2008, 53, 397-411.

(3) Balazs, E. A.; Denlinger, J. L. Viscosupplementation: a new concept in the treatment of osteoarthritis. J. Rheumatol. 1993, 20, 39.

(4) Matsuno, H.; Yudoh, K.; Kondo, M.; Goto, M.; Kimura, T. Biochemical effect of intra-articular injections of high molecular weight hyaluronate in rheumatoid arthritis patients. Inflammation Res. 1999, 48, 154-159.

(5) Takeuchi, K.; Nakazawa, M.; Yamazaki, H.; Miyagawa, Y.; Ito, T.; Ishikawa, F.; Metoki, T. Solid hyaluronic acid film and the prevention of postoperative fibrous scar formation in experimental animal eyes. Arch. Ophthalmol. 2009, 127, 460-464.

(6) Neumayer, T.; Prinz, A.; Findl, O. Effect of a new cohesive opthalmic viscosurgical device on corneal protection and intraocular pressure in small-incision cataract surgery. J. Cataract Refractive Surg. 2008, 34, 1362-1366.

(7) Brown, M. B.; Jones, S. A. Hyaluronic acid: a unique topical vehicle for the localized delivery of drugs to the skin. J. Eur. Acad. Dermatol. Venereol. 2005, 19, 308-318.

(8) Hahn, S. K.; Jelacic, S.; Maier, R. V.; Stayton, P. S.; Hoffman, A. S. Anti-inflammatory drug delivery from hyaluronic acid hydrogels. $J$. Biomater. Sci., Polym. Ed. 2004, 15, 1111-1119.

(9) Miyazaki, M.; Yuba, E.; Hayashi, H.; Harada, A.; Kono, K. Hyaluronic acid-based $\mathrm{pH}$-sensitive polymer-modified liposomes for cell-specific intracellular drug delivery systems. Bioconjugate Chem. 2018, 29, 44-55.

(10) Prosdocimi, M.; Bevilacqua, C. Exogenous hyaluronic acid and wound healing: an updated version. Panminerva Med. 2012, 54, 129135 .

(11) Ballard, K.; Cantor, A. J. Treating recalcitrant diabetic wounds with hyaluronic acid: a review of patients. Wound Manage. Prev. 2003, 49, 37-49.

(12) Abatangelo, G.; Martelli, M.; Vecchia, P. Healing of hyaluronic acid-enriched wounds: histological observations. J. Surg. Res. 1983, 35, $410-416$.

(13) Manuskiatti, W.; Maibach, H. I. Hyaluronic acid and skin: Wound healing and aging. Int. J. Dermatol. 1996, 35, 539-544.

(14) Collins, M. N.; Birkinshaw, C. Physical properties of crosslinked hyaluronic acid hydrogels. J. Mater. Sci.: Mater. Med. 2008, 19, 3335-3343.

(15) Tran, C. D.; Duri, S.; Hawkins, A. L. Recyclable synthesis, characterization, and antimicrobial activity of chitosan-based polysaccharide composite materials. J. Biomed. Mater. Res., Part A 2013, $101 A, 2248-2257$.

(16) Huang, Y.-C.; Huang, K.-Y.; Yang, B.-Y.; Ko, C.-H.; Huang, H.M. Fabrication of novel hydrogel with berberine-enriched carboxymethylcellulose and hyaluronic acid as an anti-inflammatory barrier membrane. BioMed Res. Int. 2016, 2016, No. 3640182. 
(17) Jia, Y.; Huo, M.; Huang, H.; Fu, W.; Wang, Y.; Zhang, J.; Jia, S. Preparation and characterization of bacterial cellulose/hyaluronic acid composites. Proc. Inst. Mech. Eng., Part N 2015, 229, 41-48.

(18) Credou, J.; Berthelot, T. Cellulose: from biocompatible to bioactive material. J. Mater. Chem. B 2014, 2, 4767-4788.

(19) Domingues, R. M. A.; Silva, M.; Gershovich, P.; Betta, S.; Babo, P.; Caridade, S. G.; Mano, J. F.; Motta, A.; Reis, R. L.; Gomes, M. E. Development of injectable hyaluronic acid/cellulose nanocrystals bionanocomposite hydrogels for tissue engineering applications. Bioconjugate Chem. 2015, 26, 1571-1581.

(20) Cai, Z.; Mo, X.; Zhang, K.; Fan, L.; Yin, A.; He, C. Fabrication of chitosan/silk fibroin composite nanofibers for wound dressing applications. Int. J. Mol. Sci. 2010, 11, 3529-3539.

(21) Ignatova, M.; Starbova, K.; Markova, N.; Naolova, N.; Rashkov, I. Electrospun nano-fibre mats with antibacterial properties from quarternized chitosan and poly(vinyl alcohol). Carbohydr. Res. 2006, 341, 2098-2107.

(22) Rogers, R. D.; Seddon, K. R. Chemistry. Ionic liquids-solvents of the future? Science 2003, 302, 792-793.

(23) Huddleston, J. G.; Willauer, H. D.; Swatloski, R. P.; Visser, A. E.; Rogers, R. D. Room temperature ionic liquids as novel media for "clean" liquid-liquid extraction. Chem. Commun. 1998, 1765-1766.

(24) Swatloski, R. P.; Spear, S. K.; Holbrey, J. D.; Rogers, R. D. Dissolution of cellulose with ionic liquids. J. Am. Chem. Soc. 2002, 124, 4974-4975.

(25) Tran, C. D.; Prosenc, F.; Franko, M.; Benzi, G. One-pot synthesis of biocompatible silver nanoparticle composites from cellulose and ketatin: Characterization and Antimicrobial Activity. ACS Appl. Mater. Interfaces 2016, 8, 34791-34801.

(26) Duri, S.; Harkins, A. L.; Frazier, A. J.; Tran, C. D. Composites containing fullerenes and polysaccharides: Green and facile synthesis, biocompatibility, and antimicrobial activity. ACS Sustainable Chem. Eng. 2017, 5, 5408-5417.

(27) Meng, Z.; Zheng, X.; Tang, K.; Liu, J.; Ma, Z.; Zhao, Q. Dissolution and regeneration of collagen fibers using ionic liquid. Int. J. Biol. Macromol. 2012, 51, 440-448.

(28) Isik, M.; Sardon, H.; Mecerreyes, D. Ionic liquids and cellulose: Dissolution, chemical modification and preparation of new cellulosic materials. Int. J. Mol. Sci. 2014, 15, 11922-11940.

(29) Cole, M. R.; Li, M.; Jadeja, R.; El-Zahab, B.; Hayes, D.; Hobden, J. A.; Janes, M. E.; Warner, I. M. Minimizing human infection from Escherichia coli $\mathrm{O} 157: \mathrm{H} 7$ using GUMBOS. J. Antimicrob. Chemother. 2013, 68, 1312-1318.

(30) Cole, M. R.; Hobden, J. A.; Warner, I. M. Recycling Antibiotics into GUMBOS: A New Combination Strategy to Combat MultiDrug-Resistant Bacteria. Molecules 2015, 20, 6466-6487.

(31) Warner, I. M.; El-Zahab, B.; Siraj, N. Perspectives on moving ionic liquid chemistry into the solid phase. Anal. Chem. 2014, 86, 7184-7191.

(32) Staphylococcus. https://www.foodsafety.gov/poisoning/ causes/bacteriaviruses/staphylococcus/index.html (accessed May 17, 2019).

(33) Gilli, R.; Kacurakova, M.; Mathlouthi, M.; Navarini, L.; Paoletti, S. FTIR studies of sodium hyaluronate and its oligomers in the amorphous solid phase and in aqueous solution. Carbohydr. Res. 1994, 263, 315-326.

(34) Carneiro, J.; Doll-Boscardin, P. M.; Fiorin, B. C.; Nadal, J. M.; Farago, P. V.; de Paula, J. P. Development and characterization of hyaluronic acid-lysine nanoparticles with potential as innovative dermal filling. Braz. J. Pharm. Sci. 2016, 52, 645-651.

(35) Huang, H.-C.; Chen, L.-C.; Lin, S.-B.; Hsu, C.-P.; Chen, H.-H. In situ modification of bacterial cellulose network structure by adding interfering substances during fermentation. Biosource Technol. 2010, 101, 6084-6091.

(36) Park, S.; Baker, J. O.; Himmel, M. E.; Parilla, P. A.; Johnson, D. K. Cellulose crystallinity index: measurement techniques and their impact on interpreting cellulase performance. Biotechnol. Biofuels 2010, 3, 10 .
(37) Segal, L.; Creely, J. J.; Martin, A. E.; Conrad, C. M. An empirical method for estimating the degree of crystallinity of native cellulose using the X-ray diffractometer. Text. Res. J. 1959, 29, 786794.

(38) Petroudy, S. R. D. Physical and Mechanical Properties of Natural Fibers. In Advanced High Strength Natural Fibre Composites in Construction; Fan, M.; Fu, F., Eds.; Woodhead Publishing, 2017; pp $59-83$.

(39) Wei, Y.-S.; Chen, K.-S.; Wu, L.-T. In situ synthesis of high swell ratio polyacrylic acid/silver nanocomposite hydrogels and their antimicrobial properties. J. Inorg. Biochem. 2016, 164, 17-25.

(40) Celino, A.; Freour, S.; Jacquemin, F.; Casari, P. The hygroscopic behavior of plant fibers: a review. Front. Chem. 2014, 1, No. 43.

(41) Huang, X.; Brazel, C. S. On the importance and mechanisms of burst release in matrix-controlled drug delivery systems. J. Controlled Release 2001, 73, 121-136.

(42) Setterstrom, J. A.; Tice, T. R.; Myers, W. E. Development of Encapsulated Antibiotics for Topical Administration to Wounds. In Recent Advances in Drug Delivery Systems; Anderson, J. M.; Kim, S. W., Eds.; Springer: Boston, MA, 1984.

(43) Agarwal, A.; Nelson, T. B.; Kierski, P. R.; Schurr, M. J.; Murphy, C. J.; Czuprynski, C. J.; McAnulty, J. F.; Abbott, N. L. Polymeric multilayers that localize the release of chlorhexidine from biologic wound dressings. Biomaterials 2012, 33, 6783-6792.

(44) Bauer, A. W.; Kirby, W. M. M.; Sherris, J. C.; Turck, M. Antibiotic susceptibility testing by a standardized single disk method. Am. J. Clin. Pathol. 1966, 45, 493-496.

(45) Hudzicki, J. Kirby-Bauer Disk Diffusion Susceptibility Test Protocol. https://www.asmscience.org/content/education/protocol/ protocol.3189.

(46) Cole, M. R. Chemical and Biological Evaluation of AntibioticBased Ionic Liquids and GUMBOS against Pathogenic Bacteria. LSU Doctoral Dissertations, Louisiana State University, 2012.

(47) Crowhurst, L.; Mawdsley, P. R.; Perez-Arlandis, J. M.; Salter, P. A.; Welton, T. Solvent-solute interactions in ionic liquids. Phys. Chem. Chem. Phys. 2003, 5, 2790-2794.

(48) CDC. Antimicrobial Resistance Susceptibility Testing: Disk Diffusion Susceptibility Testing, 2018. www.cdc.gov/std/gonorrhea/ lab/diskdiff.htm (accessed Aug 4, 2019). 